\title{
Sodium Polystyrene Sulfonate-Induced Gastric Pneumatosis: A Rare Side Effect
}

\author{
Neethi Dasu ${ }^{a}$ Yaser Khalid $^{b}$ Kirti Dasu $^{c}$ Lucy Joo $^{a}$ Brian Blair $^{a}$ \\ aDepartment of Gastroenterology, Jefferson Health New Jersey, Cherry Hill, NJ, USA; \\ bDepartment of Cardiology, The Wright Center for Graduate Medical Education, Scranton, \\ PA, USA; 'Department of Biomedical Sciences and Professional Studies, Drexel University, \\ Philadelphia, PA, USA
}

Keywords

Pneumatosis · Kayexalate · Gastropathy

\begin{abstract}
Kayexalate has been used in the USA since 1975 for the treatment of hyperkalemia. Prior case reports have shown that sorbitol added to kayexalate has been known to cause rare side effects of colonic necrosis. We present a unique case report of gastric pneumatosis as a complication of kayexalate.
\end{abstract}

\section{Introduction}

Sodium polystyrene sulfonate (SPS) (kayexalate) is a resin that exchanges sodium with potassium ions from the intestinal cells and then causes fecal potassium excretion [1]. Sorbitol is usually given in combination with SPS to further dissolve SPS and reduce the chances for fecal impaction and bowel obstruction [2]. Lillemoe et al. [3] reported the first side effects of sorbitol for 5 kidney transplant patients who developed colonic necrosis shortly after receiving an enema comprising 70\% sorbitol solution for the treatment of hyperkalemia.

Kayexalate-induced intestinal bowel necrosis is estimated to be $0.003-1.8 \%$, so it is an extremely rare side effect [4]. There are several proposed hypotheses on what might cause kayexalate-induced intestinal necrosis, such as blood volume lost during dialysis, hyperreninemia, elevated prostaglandin production, and vasospasm $[1,5,6]$. Patients with endstage renal disease, renal transplant, or an ileus may be more susceptible to mucosal injury. 
Fig. 1. CT scan of the chest, abdomen, and pelvis without contrast revealed a large amount of gas within the portal vein in the left lobe of the liver, in the extrahepatic portal vein, and in the wall of the stomach consistent with kayexalateinduced intestinal stomach necrosis.

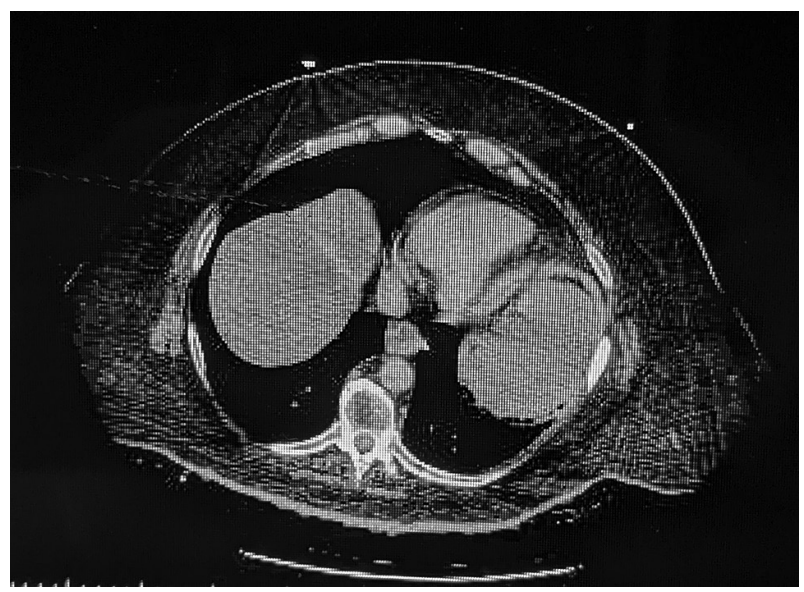

Additionally, it is postulated that the hyperosmotic load of sorbitol may directly damage intestinal mucosa, cause vasospasm of the intestinal vasculature, and exacerbate inflammation through elevated prostaglandin levels [4, 7].

We present a case of a 52-year-old male who presented with nausea and vomiting, abdominal pain, and lethargy and was diagnosed with kayexalate-induced gastric necrosis. It is an extremely rare complication and side effect that usually occurs in patients who have other risk factors such as CKD, immunosuppression, and steroid use [3, 8, 9]. There are only a few cases of kayexalate-induced intestinal bowel necrosis in the literature, and even fewer cases of gastric necrosis, making our case presentation extremely unique and rare.

\section{Case Presentation}

A 52-year-old male with a past medical history of hypertension, chronic diarrhea, hyperlipidemia, and diabetes mellitus type 2 presented from a senior living facility for lethargy, hypoglycemia, abdominal pain, and nausea and vomiting. The patient was unresponsive and markedly hypotensive. On a physical exam, the patient was somnolent with cracked mucosal membranes and subconjunctival hemorrhage of the right eye.

Initial laboratory tests revealed glucose $42 \mathrm{mg} / \mathrm{dL}(60-180 \mathrm{mg} / \mathrm{dL})$, lactate $11.5 \mathrm{mmol} / \mathrm{L}$ (0.5-1 mmol/L), potassium $6.8 \mathrm{mmol} / \mathrm{L} \mathrm{(3.6-5.2} \mathrm{mmol/L),} \mathrm{creatinine} 4.56 \mathrm{mg} / \mathrm{dL}(0.7-1.2$ $\mathrm{mg} / \mathrm{dL})$, glomerular filtration rate $14 \mathrm{~mL} / \mathrm{min} / 1.73 \mathrm{~m}^{2}\left(90-120 \mathrm{~mL} / \mathrm{min} / 1.73 \mathrm{~m}^{2}\right)$, aspartate aminotransferase 47 (5-40 U/L), alanine transaminase 72 (29-33 U/L), and CRP $9.8 \mathrm{mg} / \mathrm{dL}$ $(<10 \mathrm{mg} / \mathrm{dL})$, and kayexalate was ordered. A CT scan of the patient's chest, abdomen, and pelvis without contrast revealed a large amount of gas within the portal vein in the left lobe of the liver, in the extrahepatic portal vein, and in the wall of the stomach consistent with kayexalate-induced intestinal necrosis (Fig. 1).

A nasogastric tube was then placed for gastric decompression with interval improvement in the intestinal pneumatosis; however, coffee ground material was noted upon lavage. The patient was treated with a protonix drip, a bicarbonate drip, vasopressors, and broad-spectrum antibiotics. ACT angiography to rule out mesenteric ischemia could not be performed due to the patient's acute kidney injury. An esophagogastroduodenoscopy showed gastric mucosal injury without perforation, and biopsies were taken from the stomach and small intestine (Fig. 2). A repeat CT with IV contrast of the abdomen revealed continued necrosis but improvement in portal venous gas. The patient clinically improved. Pathology revealed reactive/chemical gastropathy with necroinflammatory debris with kayexalate crystals present (Fig. 3, 4). 

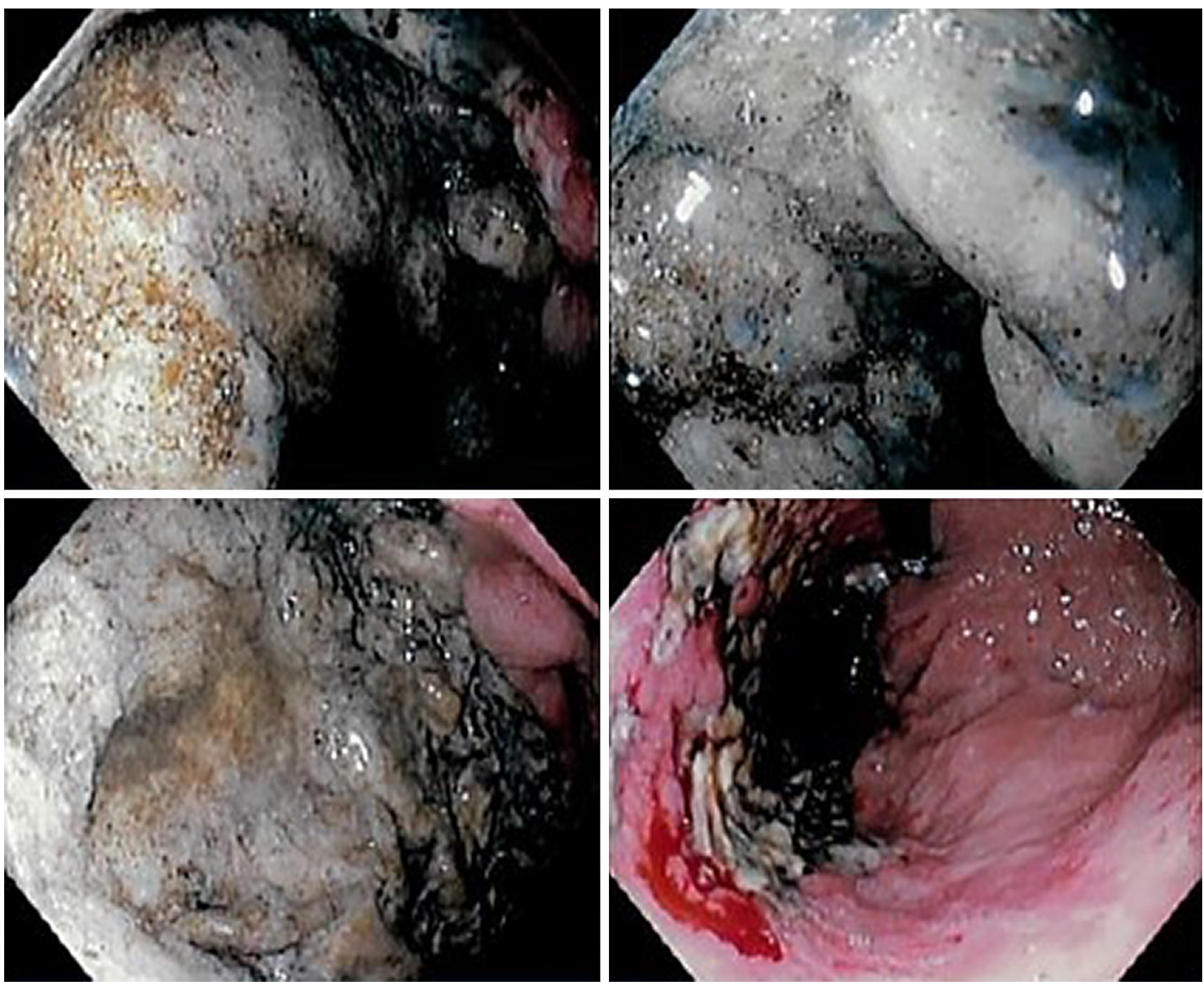

Fig. 2. EGD showing gastric mucosal injury without perforation consistent with kayexalate-induced necrosis. EGD, esophagogastroduodenoscopy.

Fig. 3. $\times 40$ magnification: pathology revealing reactive/chemical gastropathy with necroinflammatory debris with kayexalate crystals present.

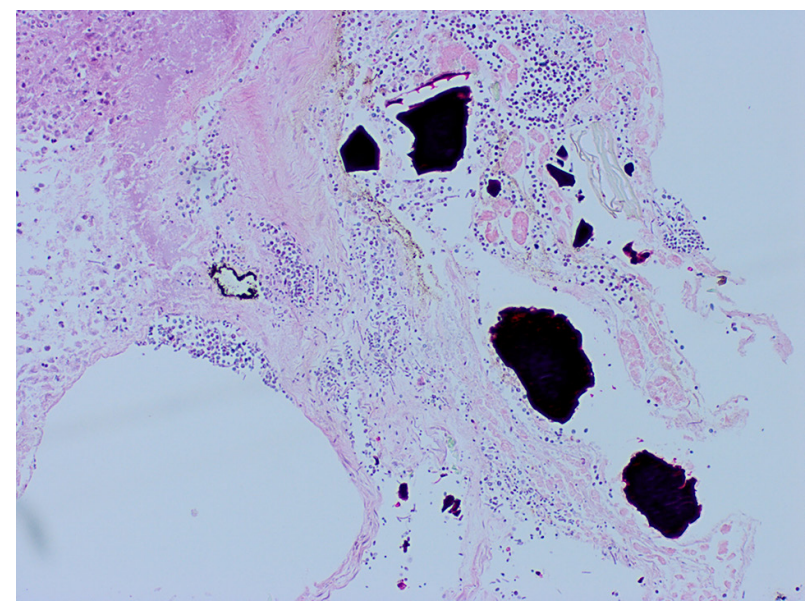

\section{Discussion}

Our case highlights an extremely rare incidence of kayexalate-induced stomach necrosis which has not been well studied previously. Prior case reports have studied kayexalate-induced intestinal bowel necrosis in patients who are relatively immunocompromised being on steroids, 
Fig. 4. $\times 100$ magnification: pathology revealing reactive/chemical gastropathy with necroinflammatory debris with kayexalate crystals present.

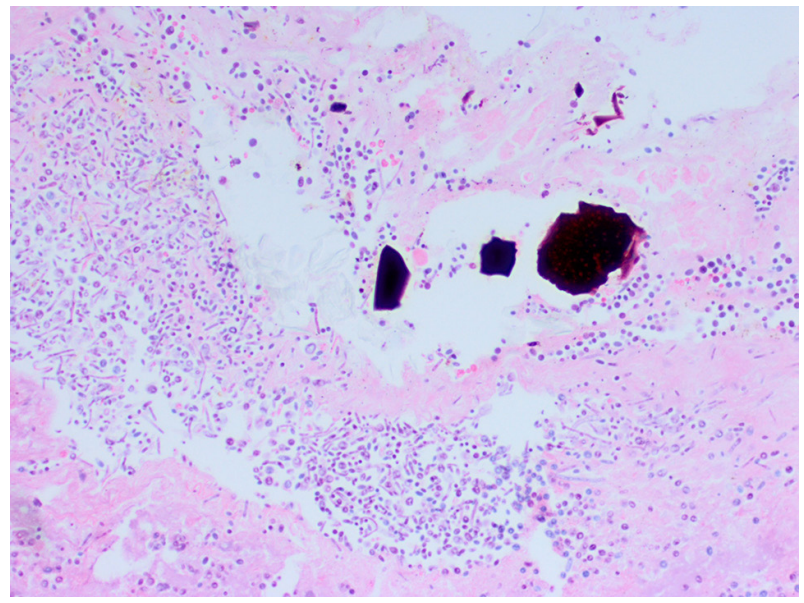

seen after surgical intervention, or receiving immunosuppressive therapy. These risk factors may predispose patients to kayexalate-induced intestinal necrosis by increasing the fragility of intestinal cells [10]. To our knowledge, this is the one of the first case reports reporting kayexalateinduced gastric necrosis in a relatively healthy 52 -year-old male. The case further proves that upper gastrointestinal damage associated with kayexalate is relatively benign and is shown to have less mortality than lower GI symptoms as seen in our patient $[7,11]$. The spectrum of how kayexalate affects the GI tract needs to further be studied as the pathophysiology is yet unknown.

Furthermore, animal models have depicted that a lesser concentration of sorbitol of approximately $33 \%$ has decreased the rate of developing colonic necrosis following administration of kayexalate with sorbitol, but this risk still exists regardless of the level of sorbitol given with kayexalate. This is important in our case because kayexalate at our hospital system contains 33\% sorbitol, yet there was still significant injury from kayexalate for our patient. Even though the number needed to harm in kayexalate is 1 in 1,395, practitioners should use kayexalate with caution. Alternative medications, such as loop diuretics, or using hemodialysis to help excrete the potassium are useful alternatives.

Most case reports that report kayexalate-induced intestinal pneumatosis have pre-existing renal failure, irrespective of etiology. This is an important clinical correlation to consider and needs further research to further elucidate whether kayexalate is safe for this patient population. The exact mechanism of why this patient population has a higher susceptibility to kayexalateinduced intestinal side effects is unknown. It is hypothesized that patients with renal disease have an elevated level of renin which can predispose these patients to nonocclusive mesenteric ischemia via angiotensin-mediated vasoconstriction. In combination with the osmotic effect of sorbitol, the intestinal vasculature becomes prone to injury which can lead to the development of colonic necrosis and eventual air formation in the gut wall. Additional risk factors are uremia, hypovolemia, PVD, and immunosuppressive therapy such as steroids $[1,3,8,12]$.

Finally, kayexalate-induced intestinal necrosis usually occurs after $3 \mathrm{~h}$ of administration and can occur up to 11 days following administration. The presence of crystals on biopsy as a mosaic pattern on the H\&E stain is pathognomonic.

\section{Conclusion}

Our case report highlights that further complications seen in the GI tract can still be observed, and more data are needed to determine the rate and incidence of these complications while administering kayexalate.

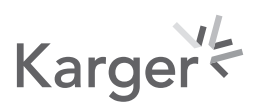




\section{Acknowledgments}

We would like to thank the patient for allowing us to share the details of his clinical history. We would also like to express our appreciation to the Pathology Department at Jefferson Health NJ, USA, for providing us with the pathology slides.

\section{Statement of Ethics}

Written informed consent was obtained from the patient for publication of this case report and any accompanying images. This study protocol was reviewed, and the need for approval was not required in accordance with local guidelines.

\section{Conflict of Interest Statement}

The authors have no conflicts of interest to declare.

\section{Funding Sources}

No funding was received for this article.

\section{Author Contributions}

N.D. and Y.K. were responsible for the study concept and design, data collection, and drafting of the manuscript. N.D., K.D. and Y.K. edited the contents and grammar of the manuscript. L.J. and B.B. supervised the entirety of the study.

\section{Data Availability Statement}

All data generated or analyzed during this study are included in this article and its online suppl. Material Files (for all online suppl. material, see www.karger.com/doi/10.1159/000518929). Further enquiries can be directed to the corresponding author.

\section{References}

1 McGowan CE, Saha S, Chu G, Resnick MB, Moss SF. Intestinal necrosis due to sodium polystyrene sulfonate (Kayexalate) in sorbitol. South Med J. 2009;102(5):493-7.

2 Bomback AS, Woosley JT, Kshirsagar AV. Colonic necrosis due to sodium polystyrene sulfate (Kayexalate). Am J Emerg Med. 2009;27(6):753-2.

3 Lillemoe KD, Romolo JL, Hamilton SR, Pennington LR, Burdick JF, Williams GM. Intestinal necrosis due to sodium polystyrene (Kayexalate) in sorbitol enemas: clinical and experimental support for the hypothesis. Surgery. 1987;101(3):267-72.

4 Patel S, Arnold CA, Gray DMI. A case of sodium polystyrene sulfonate (Kayexalate)-induced bowel ischemia: a reminder of adverse effects of a common medication: 2501. Off J Am Coll Gastroenterol ACG. 2017;112:S1365.

5 Singhania N, Al-Odat R, Singh AK, Al-Rabadi L. Intestinal necrosis after co-administration of sodium polystyrene sulfonate and activated charcoal. Clin Case Rep. 2020;8(4):722-4.

\section{Karger'}


6 Watson MA, Baker TP, Nguyen A, Sebastianelli ME, Stewart HL, Oliver DK, et al. Association of prescription of oral sodium polystyrene sulfonate with sorbitol in an inpatient setting with colonic necrosis: a Retrospective Cohort Study. Am J Kidney Dis. 2012;60(3):409-16.

7 Rashid A, Hamilton SR. Necrosis of the gastrointestinal tract in uremic patients as a result of sodium polystyrene sulfonate (Kayexalate) in sorbitol: an underrecognized condition. Am J Surg Pathol. 1997;21(1):60-9.

8 Harel Z, Harel S, Shah PS, Wald R, Perl J, Bell CM. Gastrointestinal adverse events with sodium polystyrene sulfonate (Kayexalate) use: a systematic review. Am J Med. 2013;126(3):264.e9-24.

9 Gardiner GW. Kayexalate (sodium polystyrene sulphonate) in sorbitol associated with intestinal necrosis in uremic patients. Can J Gastroenterol. 1997;11(7):573-7.

10 Hajjar R, Sebajang H, Schwenter F, Mercier F. Sodium polystyrene sulfonate crystals in the gastric wall of a patient with upper gastrointestinal bleeding and gastric perforation: an incidental finding or a pathogenic factor? J Surg Case Rep. 2018;2018(6):rjy138.

11 Abraham SC, Bhagavan BS, Lee LA, Rashid A, Wu TT. Upper gastrointestinal tract injury in patients receiving kayexalate (sodium polystyrene sulfonate) in sorbitol: clinical, endoscopic, and histopathologic findings. Am J Surg Pathol. 2001;25(5):637-44.

12 Dardik A, Moesinger RC, Efron G, Barbul A, Harrison MG. Acute abdomen with colonic necrosis induced by Kayexalate-sorbitol. South Med J. 2000;93(5):511-3. 